\title{
Outcomes of Nerve Transfer versus Nerve Graft in Ulnar Nerve Laceration
}

\author{
K. Ming Chan, Jaret L. Olson, Michael Morhart, Tan Lin, Regan Guilfoyle
}

Can J Neurol Sci. 2012; 39: 242-244

\section{BACKGROUND}

Because of the long distance between the injury site and the target hand muscles, functional recovery following proximal ulnar nerve injury is often poor. In addition to progressive decline of trophic support for nerve regrowth, muscle atrophy also plays a major role. Even with primary surgical repair, functional recovery of the intrinsic hand muscles is often unsatisfactory, resulting in semi claw hand deformity, marked pinch and grip weakness. ${ }^{1}$

Nerve grafting using sensory donor nerves, popularized in the 1970 s, is one way to bridge the gap and to provide a conduit for the advancing growth cone to extend towards the target muscles. Because the results have not been consistently successful, nerve transfers to the distal stump using redundant motor branches to neighboring muscles have been proposed as a viable alternative. However, how conducive these options are in attracting the regenerating nerve fibres has not been directly compared. In this case report, we present a patient in whom both sensory nerve grafts and distal nerve transfer were used at the same site to repair a transected ulnar nerve in the forearm. This afforded us a unique opportunity of directly comparing the relative success of each method in allowing motor reinnervation to the intrinsic hand muscles. Using electrophysiological methods to follow the nerve regeneration over three years, we found a major discrepancy in the proportions of regenerating motor nerve fibres that successfully reached the target muscles from each source.

\section{Case Report}

This otherwise healthy 44-year-old right-handed power engineer was injured when the spinning wheel of a grinder broke off and lacerated the volar aspect of the left distal forearm. On physical examination the patient did not have any movement of the ulnar innervated intrinsic hand muscles. In contrast, the median nerve innervated muscles were normal. He also had normal function of the long finger and wrist flexors including flexor carpi ulnaris and digitorum profundus to the 4th and 5th digits when examined five weeks after his injury. Sensory examination revealed reduced pinprick, touch, vibration and temperature sensations in the ulnar innervated territory in his left hand. Stretch reflexes were normal and symmetrical at $2+$. The right arm was completely symptom free.

Although the cut flexor carpi ulnaris and flexor digitorum superficialis tendons to the 4 th and 5 th digits as well as the ulnar artery could be repaired immediately after injury, the ulnar nerve was not amenable to direct resuturing as there was a gap between the proximal and distal nerve stump. At a separate operation performed six weeks after injury, the laceration scar in the mid forearm was reopened and the transected ulnar nerve ends identified. The proximal and distal stumps were carefully trimmed back by microsurgery until viable intact nerve fascicles were found and confirmed by frozen sections. This left a $2 \mathrm{~cm}$ gap that was bridged using five sural autogenous grafts secured with Tisseel glue. The flexor digitorum superficialis muscle was then lifted and the anterior interosseous nerve (AIN) exposed and followed to the proximal border of the pronator quadratus muscle. A neurolysis was performed and the terminal motor branches to the muscle were transected and attached onto the distal ulnar nerve stump in an end-to-end fashion. No attempt was made to match the fascicles in the distal nerve stump. The wrist was moved through the full range of motion to ensure that the coaptation was not under tension at any point.

\section{RESULTS}

During follow up evaluations appropriately every six months, motor conduction studies of the ulnar nerve were done by recording over the hypothenar muscles and the first dorsal interossei (FDI) muscles. Sensory nerve conduction studies were also done by recording over the digital nerve in the 5th digit. While no motor or sensory response could be elicited at the initial assessments, at 18 months post-operation, a maximal compound muscle action potential with a negative-peak amplitude of $2.69 \mathrm{mV}$ (compared to $7.10 \mathrm{mV}$ on the uninjured side) could be elicited in the hypothenar muscles by stimulating the ulnar nerve just distal to the repair site. The majority of the regenerated motor axons were from the AIN since a large maximum compound muscle action potential could be obtained by stimulating the median nerve at the elbow (Figure). In contrast, stimulating the ulnar nerve over the proximal nerve stump only elicited a small response at $0.82 \mathrm{mV}$. The same trend was also observed in the FDI muscle. While the compound muscle action potential (CMAP) obtained from the FDI muscle

From the Division of Physical Medicine and Rehabilitation (KMC), Centre for Neuroscience (KMC, TL), Department of Plastic Surgery (JLO, MM, RG), University of Alberta, Edmonton, Alberta, Canada.

Received August 23, 2011. Final Revisions Submitted October 27, 2012. Correspondence to: K. Ming Chan, 5005C Katz Group Centre for Pharmacy and Health Research, University of Alberta, Edmonton, Alberta, T6G 1E2, Canada. 


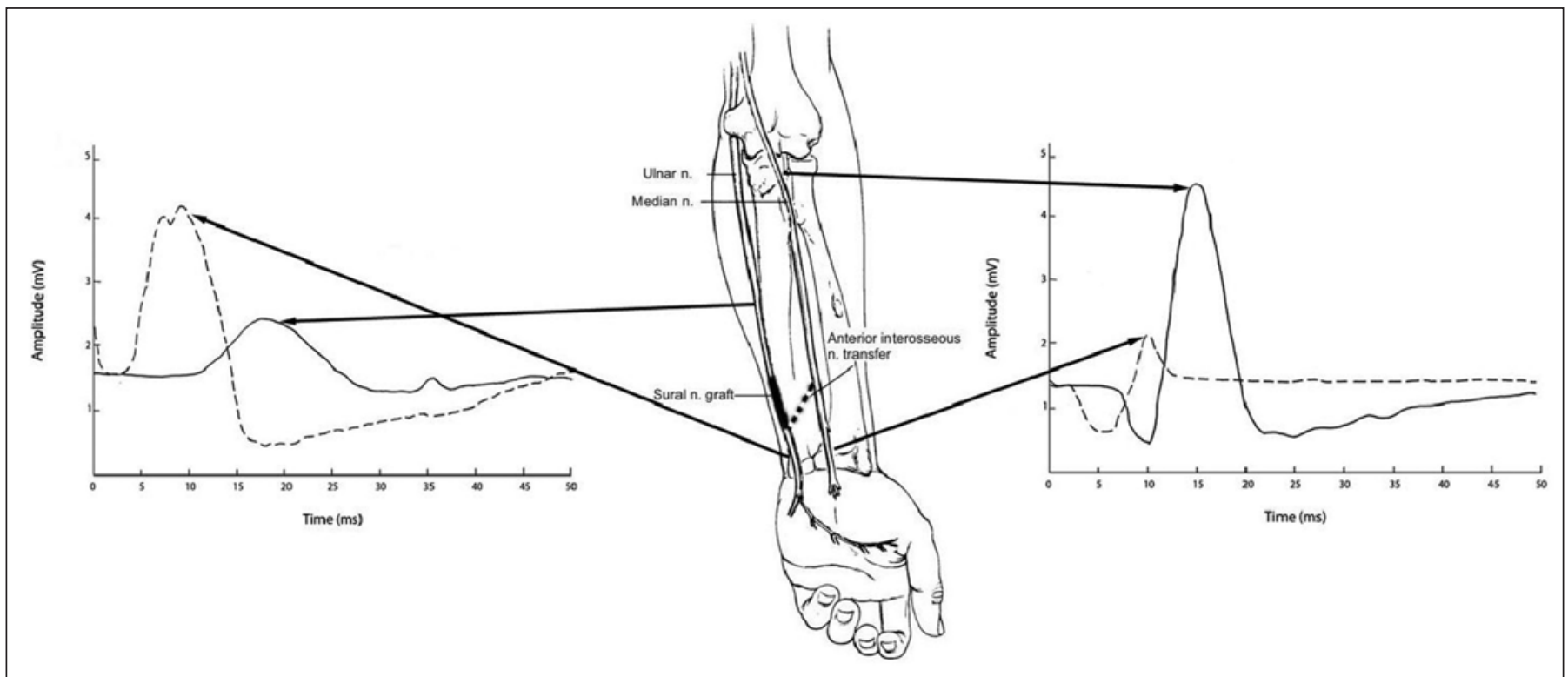

Figure: Reinnervation of the hypothenar muscles. This study done at 18 months after surgery shows the relative contribution from the proximal ulnar nerve stump regenerating through the sural nerve graft (in black on the forearm figure) compared to that from the anterior interosseous nerve (dash line). Amplitude of the maximum combined muscle action potential elicited from the ulnar nerve in the proximal forearm (solid line on left panel) was substantially smaller than that elicited at the wrist (dash line). The opposite proportional difference was found when stimulating the median nerve (right panel) indicating that the majority of the regenerated motor axons originated from the transferred anterior interosseous nerve.

by stimulating the distal ulnar nerve stump was $0.76 \mathrm{mV}$ (compared to $5.12 \mathrm{mV}$ on the uninjured side), the vast majority of it was through regenerated motor axons in the AIN because stimulation of the proximal ulnar stump only accounted for 0.10 $\mathrm{mV}$. These results indicated that over $70 \%$ of the regenerated axons in the case of the hypothenar muscles and over $90 \%$ in the FDI muscle came from the AIN nerve. The relative contributions from the transferred AIN nerve and the proximal ulnar nerve stump remained similar during follow up over the subsequent 18 months. At three years, the CMAP amplitude of the hypothenar muscles was $6.80 \mathrm{mV}$ with only a $2.1 \mathrm{mV}$ contribution from the ulnar nerve.

Reinnervation of the intrinsic hand muscles resulted in substantial functional improvements. The grip strength, measured by using a Jamar dynamometer, increased from $9.7 \mathrm{~kg}$ at baseline to $35.1 \mathrm{~kg}$ at 36 months, an almost three fold increase. The key pinch strength increased from $2.2 \mathrm{~kg}$ to $4.4 \mathrm{~kg}$ and the tip-to-tip strength went from $2.1 \mathrm{~kg}$ to $5.2 \mathrm{~kg}$. On manual muscle testing, abduction strength of the 2nd digit was at MRC grade 4+ while abduction of the 5 th digit was at grade 5 . Sensory function was evaluated through 2 point discrimination over the pulp of the 5 th digit. While the patient was able to detect a $4 \mathrm{~mm}$ distance of separation in the right hand, he was unable to reliably detect the 2 points in the left hand even when the stimuli were $20 \mathrm{~mm}$ apart. Fortunately, since fine touch sensations on the median nerve innervated digits necessary for dexterous movements were intact, he did not perceive any disability associated with the reduced sensation in the ulnar nerve territory.

\section{Discussion}

To our knowledge, this is the first reported case that directly quantified the difference in nerve regeneration through a transferred nerve as opposed to nerve grafts at the same site. We found that regeneration from the transferred terminal branch from the AIN was much more robust than that through the sural nerve grafts.

Although autologous nerve graft was introduced over a century ago, the extent of regeneration through the graft and degree of functional recovery in many cases remain disappointing. ${ }^{2}$ Indeed, despite the popularization of the procedure since the 1970 s, there has not been any major change in clinical outcomes in the last 35 years. For example, in a large retrospective study of 407 cases of surgically repaired ulnar nerve trauma caused by gunshot and shrapnel, Secer et al reported an overall good recovery ( $\geq$ grade 4 MRC motor scale) in $50 \%$ of those injured in the distal forearm. ${ }^{3}$ However, the subgroup that required end-to-end interfascicular coaptation with sural grafts did substantially worse. Potential reasons for this include the length of graft that the regenerating axons needs to traverse before reaching the distal stump, the existence of two suture sites where the crossing can be very slow and, lastly, the use of sensory nerve graft that can inhibit motor nerve growth. ${ }^{2}$ In contrast, distal nerve transfers can potentially circumvent some of these obstacles as there is only one suture site and that the donor nerve contains a high percentage of motor axons. Since it has been shown that certain fascicles in the ulnar nerve have higher motor nerve fibre content than others, ${ }^{4}$ one potential alternative explanation for our findings is that the sural nerve grafts might have been inadvertently anastomosed to areas away from those fascicles and were therefore at a disadvantage when trying to find a motor path. However, that seems a highly unlikely explanation for several reasons. First, the method of coaptation using Tisseel glue is highly non-selective and does not allow for precise fascicular matching. Second, in terms of 
surface diameter, the AIN donor is relatively small compared to that of the sural nerve grafts. Therefore, probabilistically the sural nerve grafts had an advantage over the AIN by having a larger area of contact with the fascicles in the distal nerve stump. However, there are limitations to distal nerve transfers. First, it is only feasible when there are viable nerves in the immediate vicinity that can serve as donors. Second, the denervation of neighboring muscles can result in weakness. Fortunately, in the case of forearm pronation, the pronator teres muscle is a prime mover and is able to compensate for the loss of the pronator quadratus muscle. Lastly, for optimal reinnervation, the axon numbers in the donor nerve should be at least equal or greater than that in the recipient nerve. In fresh cadaver studies, the AIN motor branches to the pronator quadratus muscle has been shown to have axon numbers that closely matches that of the ulnar motor branch. 5

\section{Conclusion}

We report a unique case where both sensory nerve grafts and a distal nerve transfer were done at the same location to repair a high ulnar nerve cut. The terminal motor branch transfer from a neighboring muscle appeared to be much more efficacious than the sural nerve grafts. This observation is of potential importance when considering nerve repair options.

\section{REFERENCES}

1. Mailander P, Berger A, Schaller E, Ruhe K. Results of primary nerve repair in the upper extremity. Microsurgery. 1989; 10: 147-50.

2. Nichols CM, Brenner MJ, Fox IK, et al. Effects of motor versus sensory nerve grafts on peripheral nerve regeneration. Exp Neurol. 2004; 190:347-55.

3. Secer HI, Daneyemez M, Gonul E, Izci Y. Surgical repair of ulnar nerve lesions caused by gunshot and shrapnel: results in 407 lesions. J Neurosurg. 2007; 107:776-83.

4. Chow JA, Van Beek AL, Meyer DL, Johnson MC. Surgical significance of the motor fascicular group of the ulnar nerve in the forearm. J Hand Surg Am. 1985; 10:867-72.

5. Ustun ME, Ogun TC, Buyukmumcu M, Salbacak A. Selective restoration of motor function in the ulnar nerve by transfer of the anterior interosseous nerve. An anatomical feasibility study. J Bone Joint Surg Am. 2001; 83-A:549-52. 